\title{
Study on the diagnostic system of scoliosis by using infrared camera
}

\author{
Jin-hyoung Jeong ${ }^{\mathrm{a}}$, Eun-jeong Park ${ }^{\mathrm{b}}$, Chang-ok Cho ${ }^{\mathrm{c}}$, Yoon-jeong Kim ${ }^{\mathrm{d}}$ and Sang-sik Lee ${ }^{\mathrm{a}}{ }^{*}$ \\ ${ }^{a}$ Department of Biomedical Engineering, Catholic Kwandong University, Gangneung, Korea \\ ${ }^{b}$ Kangwon National University School of Medicine, Chuncheon, Korea \\ ${ }^{c}$ Korea Paralympic Committee, Seoul, Korea \\ ${ }^{d}$ Department of Social Welfare, Catholic Kwandong University, Gangneung, Korea
}

\begin{abstract}
In this study, the radiation generated in the diagnosis of scoliosis, to solve the problems by using an infrared camera and an optical marker system that can diagnose scoliosis developed. System developed by the infrared camera attached to the optical spinal curvature is recognized as a marker to shoot the angle between the two optical markers are measured. Measurement of angle, we used the Cobb's Angle method used in the diagnosis of spinal scoliosis. We developed a software to be able to output to the screen using an infrared camera to diagnose spinal scoliosis. Software is composed of camera output unit was manufactured in Labview, angle measurement unit, in Cobb's Angle measurement unit .In the future, kyphosis, Hallux Valgus, such as the diagnosis of orthopedic disorders that require the use of a diagnostic system is expected case.
\end{abstract}

Keywords: Scoliosis, optical marker, Cobb's angle, infrared camera, spine

\section{Introduction}

Scoliosis is anatomical spine the axis of the middle from the sides rather than only by deformation date on curved or coronal plane laterally, the rotation of the vertebral be entrained in thalamus surface is loss settlement bendable condition in which is a three-dimensional deformation. It is a spinal disorder that can result in significant long-term and be compressed to dysfunction when deformation is severe and causes little pain or apparent problem. About $85 \%$ to $90 \%$ of scoliosis is classified particularly into Pentagon scoliosis that it can't determine the cause daily life type and posture, as trauma or age increases, also be affected, but prophylaxis is no what is known to date, early detection is a very important disease. Especially scoliosis is, by providing the non-flexible physical activity to accompany the stiffness and pain is not only good appearance. It can cause many obstacles to the correct daily life youth and spine side of the elderly continuous treatment and scoliosis prevention and early diagnosis is important [1-3].

The equipment currently diagnosing scoliosis, physical checker, Moire surface measuring instrument, and that the spine side maturity, several methods, such as radiography, but the

\footnotetext{
* Address for correspondence: Sangsik Lee, Department of Biomedical Engineering, Catholic Kwandong University, Gang neung, Korea. Tel.: +82.33.649.7584; Fax: +82 33647 7539; E-mail:lsskyj@cku.ac.kr.
}

0959-2989/15/\$35.00 @ 2015 - IOS Press and the authors. 
Table 1

Camera specifications

\begin{tabular}{ll}
\hline Image sensor & $1 / 3 "$ progressive scan CMOS \\
\hline Resolution & $752 \times 480$ \\
Interface & 6 pin IEEE-1394b \\
Frame rate & $752 \times 480$ at $60 \mathrm{FPS} \cdot 320 \times 240$ at $112 \mathrm{FPS}$ \\
Power & $8 \sim 30 \mathrm{~V}$ \\
Dimension & $24.4 \times 44 \times 34 \mathrm{~mm}$ \\
\hline
\end{tabular}

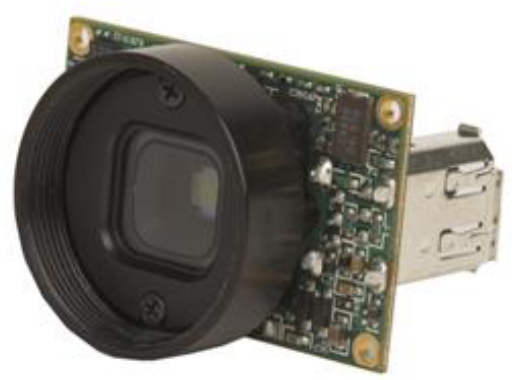

Fig. 1. Alignment camera (USA).

radiographic method to accurately measure the curvature of scoliosis it is there can be equipment [4] However, in the case of radiography, certain disadvantages can be rows negative effect by the radiation exposure is reached. Also it is inefficient in price specific surface.

In this study, it is desired to study the system that can be to try to solve the problem that occurs when radiation imaging using a general infrared camera to diagnose the degree of scoliosis curvature $[5,6]$.

\section{Materials and methods}

\subsection{Infrared camera}

In this study, the camera used is a alignment camera, that are equipped with various CMOS sensor from the same 0.3 Mega as Figure 1 to 1.3 Mega. Camera spec's the following Table 1 and Figure 1.

\subsection{Infrared illumination and lighting controller}

Infrared illumination is a ring-shaped illumination, such as the following Figure 1 (LV-DRT-90R-IR, USA). Infrared wavelength is $850 \mathrm{~nm}$, the total number of LED is 204 pieces [7].

Lighting controller for the infrared illumination brightness adjustment (LVS-PN22IR, USA) and is he the next Figures 2 and 3.

\subsection{Lens and filter}

The camera lens using the same $5 \mathrm{~mm}$ lens (H0514-MP2, USA) and Figures 4 and 5 (pass filters), using a filter that passes the more infrared $850 \mathrm{~nm}$ (BP 850, USA). 


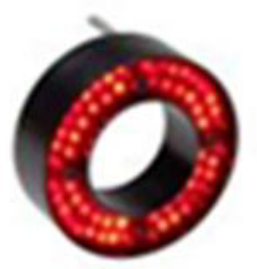

Fig. 2. Infrared illumination.

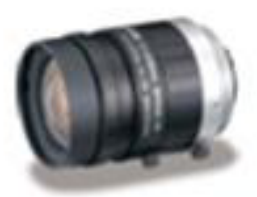

Fig. 4. Camera lens.

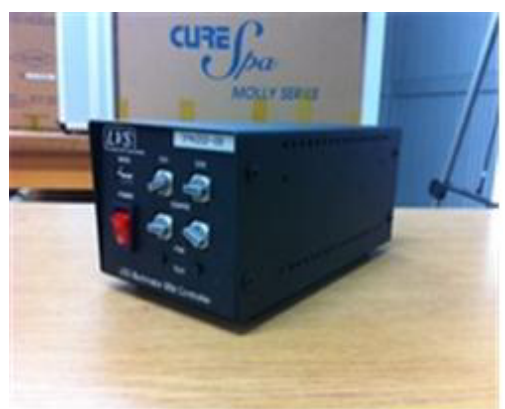

Fig. 3. Infrared controller.

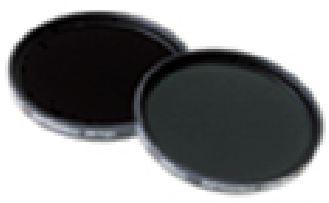

Fig. 5. Filter.

\subsection{Scoliosis diagnostic software and Cobb's angle}

Program for the diagnosis of scoliosis, use Labview 8.5, was constructed software program. Below Figure 6, scoliosis, an entire program, program for the diagnosis of scoliosis, the output of the camera, the ratio measurement unit of the size of the left and right, the angle measuring unit, Cobb's Angle measuring unit, is constituted by Cobb's Angle description section.

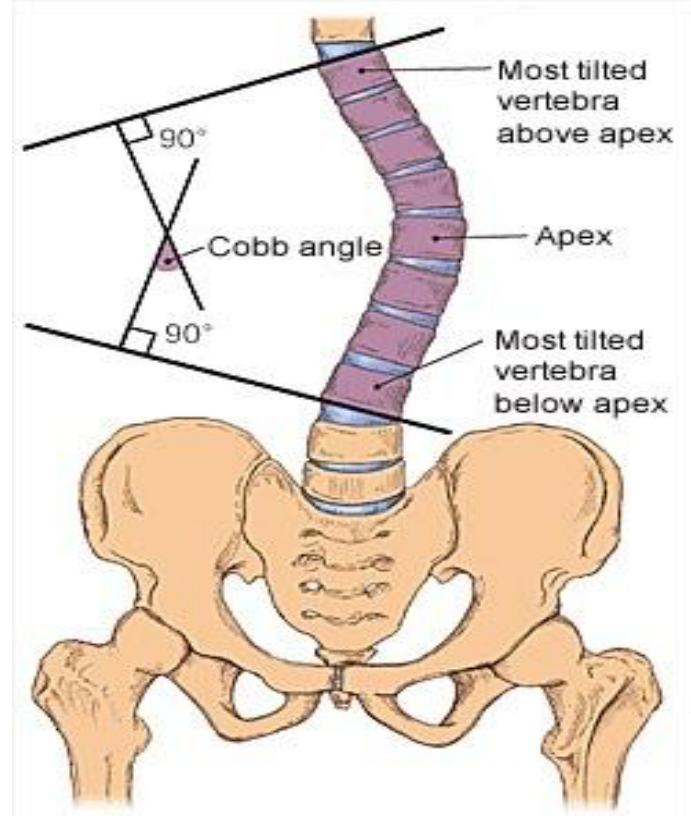

Fig. 6. Cobb’s angle measurement. 


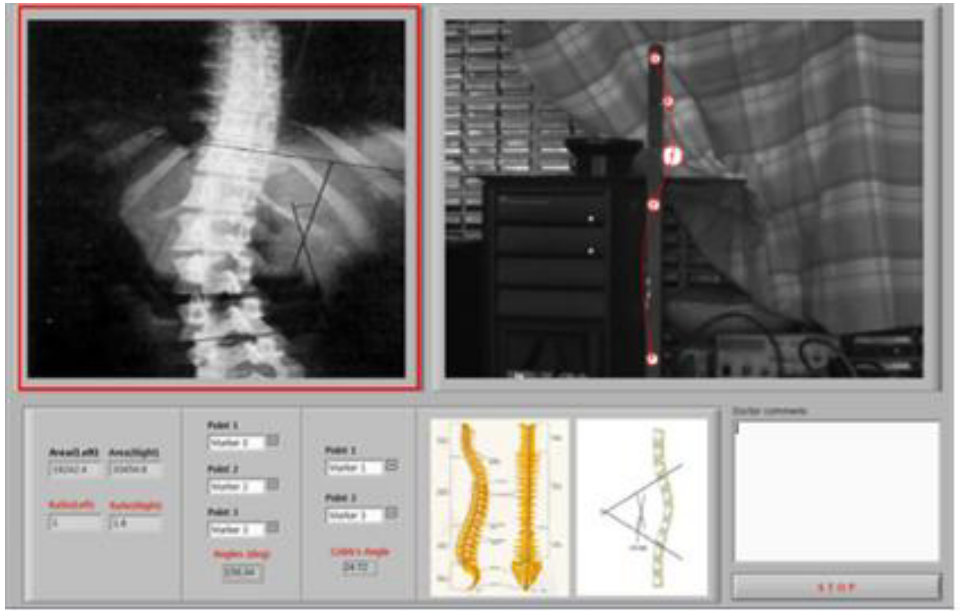

Fig. 7. Full screen program.

Cobb's Angle a measurement used for evaluation of curves in scoliosis on an AP radiographic projection of the spine. When assessing a curve the apical vertebra is first identified; this is the most likely displaced and rotated vertebra with the least tilted end plate. The end/transitional vertebra are then identified through the curve above and below. The end vertebra are the most superior and inferior vertebra which are least displaced and rotated and have the maximally tilted end plate. A line is drawn along the superior end plate of the superior end vertebra and a second line drawn along the inferior end plate of the inferior end vertebra. If the end plates are indistinct the line may be drawn through the pedicles. The angle between these two lines (or lines drawn perpendicular to them) is measured as the Cobb angle.

In S-shaped scoliosis where there are two contiguous curves the lower end vertebra of the upper curve will represent the upper end vertebra of the lower curve. Because the Cobb angle reflects curvature only in a single plane and fails to account for vertebral rotation it may not accurately demonstrate the severity of three dimensional spinal deformity. As a general rule a Cobb angle of 10 is regarded as a minimum angulation to define scoliosis.

The output of the camera body other than the optical marker is not recognized, the optical markers would be captured by the red frame. Markers, in turn, are displayed in 0,1 , and 2 in the order.

Left. Measuring unit the ratio of the size of the right Area (Left)/ Area (Right), it displays the size of the left and right based on the coordinate values of the top marker.

Optical marker of angle measurement unit is I choose the selected point. Selecting the marker points $1,2,3$, so that measuring the angle between the three markers in the company Angle.

When Cobb's Angle measurement unit to the input marker by select multiple parts that seem to scoliosis, Cobb's Angle measurements of the two marker points part comes out [8-10].

Figure 7 shows the experimental scene of a program created with LabVIEW.

\section{Experimental methodology}

The existing scoliosis measurement method is to measure the degree spine side bay curved X-ray image of a patient by applying the Cobb's Angle. First, Cobb's Angle, depending on the angle, as shown in Table 2 below, is divided into four ranks. Usually it can be seen from $8^{\circ}$ to $10^{\circ}$ normal. 
Table 2

Cobb's angle curvature

\begin{tabular}{ll}
\hline Stage & Cobb's angle \\
\hline Normal & $0^{\circ}$ \\
Mild & $1^{\circ} \sim 19^{\circ}$ \\
Moderate & $20^{\circ} \sim 39^{\circ}$ \\
Severe & $40^{\circ} \sim$ \\
\hline
\end{tabular}

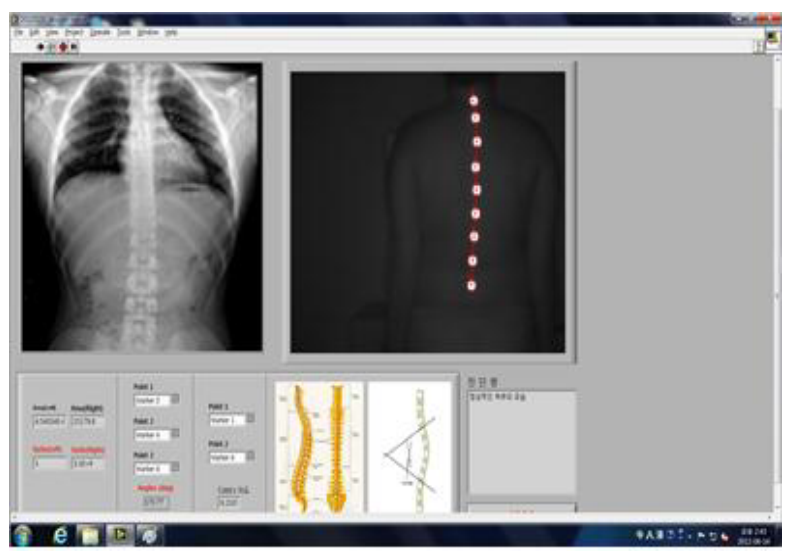

Fig. 8. Measuring Photos.

Table 3

17 people five times the measured value $\mathrm{M}$

\begin{tabular}{llllll}
\hline & Measured value 1 & Measured value 2 & Measured value 3 & Measured value 4 & Measured value 5 \\
\hline m 1 & 26.529 & 22.177 & 25.918 & 23.794 & 22.772 \\
m 2 & 3.6803 & 4.1005 & 5.1498 & 4.4106 & 4.2335 \\
m 3 & 4.4954 & 4.6937 & 4.4992 & 4.2197 & 4.3112 \\
m 4 & 8.8169 & 6.3423 & 6.5497 & 6.9297 & 7.5267 \\
m 5 & 11.251 & 11.028 & 10.814 & 11.288 & 11.36 \\
m 6 & 2.352 & 2.4958 & 2.3854 & 1.5588 & 2.1981 \\
m 7 & 7.9871 & 7.2702 & 7.7794 & 7.5318 & 7.8253 \\
m 8 & 4.8549 & 5.4349 & 5.2194 & 5.6992 & 5.4086 \\
m 9 & 6.4219 & 5.6602 & 7.1453 & 69.847 & 9.0786 \\
m 10 & 6.2835 & 7.179 & 8.8123 & 8.8123 & 7.7318 \\
m 11 & 11.607 & 9.2652 & 9.5152 & 9.7139 & 8.9803 \\
m 12 & 8.1994 & 13.759 & 9.358 & 5.7775 & 3.7438 \\
m 13 & 9.6946 & 5.7835 & 4.4172 & 2.1984 & 2.0673 \\
m 14 & 5.2154 & 5.138 & 5.0511 & 5.2535 & 4.8966 \\
m 15 & 6.8491 & 7.3511 & 6.9728 & 7.1588 & 6.7728 \\
m 16 & 2.0035 & 4.6544 & 1.761 & 5.8735 & 3.6187 \\
m 17 & 9.2187 & 9.8327 & 10.442 & 10.111 & 10.399 \\
\hline
\end{tabular}

In this study, was taken image based diagnostic methods for measuring the curvature of the spine side bay. By attaching the optical markers according to the spine as the lower 7 to detect the degree of curvature, to shoot an optical marker infrared camera. Only markers in side shown on the camera output unit to measure the angle at Cobb's Angle measuring part of the three number selected by the selection at an angle measuring unit markers of the place marker [11, 12]. 
Table 4

13 people five times more than the measure

\begin{tabular}{llllll}
\hline & Measured value 1 & Measured value 2 & Measured value 3 & Measured value 4 & Measured value 5 \\
\hline f 1 & 9.2514 & 9.334 & 9.5672 & 9.8868 & 9.4263 \\
f 2 & 10.902 & 10.613 & 10.773 & 9.6732 & 10.997 \\
f 3 & 8.4519 & 9.6156 & 9.5512 & 9.7422 & 9.794 \\
f 4 & 12.149 & 12.345 & 12.01 & 12.776 & 11.979 \\
f 5 & 15.973 & 16.178 & 15.198 & 15.928 & 13.872 \\
f 6 & 16.376 & 16.657 & 16.238 & 17.046 & 16.541 \\
f 7 & 15.047 & 14.645 & 14.998 & 15.879 & 15.338 \\
f 8 & 13.08 & 13.23 & 12.993 & 12.79 & 12.64 \\
f 9 & 8.1293 & 10.057 & 9.4255 & 9.3129 & 9.4838 \\
f 10 & 14.257 & 14.474 & 15.447 & 16.212 & 45.213 \\
f 11 & 14.708 & 14.125 & 14.508 & 14.735 & 14.455 \\
f 12 & 4.3993 & 4.6281 & 4.9689 & 4.0078 & 3.896 \\
f 13 & 19.417 & 9.0242 & 9.2841 & 9.467 & 9.0288 \\
\hline
\end{tabular}

Table 5

Male, female subjects the mean and standard deviation, standard error

\begin{tabular}{llll}
\hline & Average & Standard Deviation & Standard error \\
\hline Male & 0.42088 & 0.415289 & 0.185723 \\
Female & 0.184244 & 0.090981 & 0.40688 \\
\hline
\end{tabular}

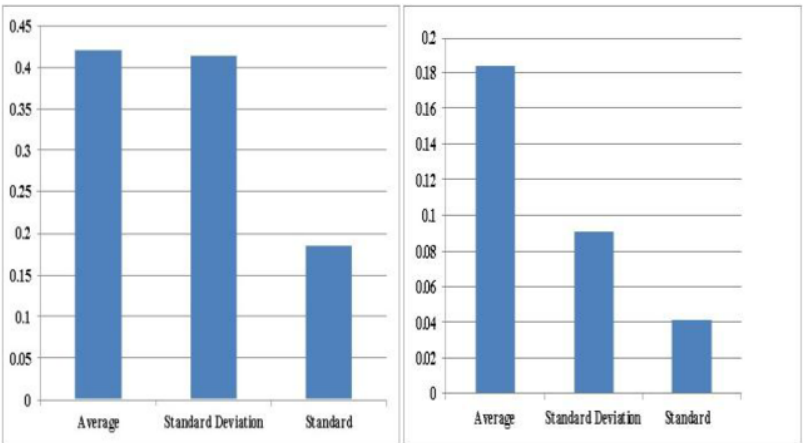

Fig. 9. All of the average, standard deviation, standard deviation.

\section{Result and discussion}

In order to derive the results of the value of the present study, people of Catholic Kwandong University, was diagnosed spinal scoliosis to target a total of 30 women 13 and 17 man people. It was measured five times for each subject to determine the standard deviation and standard error, respectively. Figure 8 is a drive image of diagnostic system of scoliosis. Was measured by undressing the upper body of the subject for the diagnosis of scoliosis.

Scoliosis diagnostic value of measuring a man and woman in this way are shown in Table 3 and 4.

Results of measurement in this algorithm, the overall standard deviation men 0.415289 , the standard error is found in 0.185723 overall standard deviation women 0.090981 , the standard error was 0.040688. Table 5 below lists the mean and standard deviation of the whole of the experiment and the value of the standard error. 
The results of the experiment, 17 men, intended for subjects 13 females, a result of measuring the Cobb's Angle value that is diagnosis of scoliosis, 30 hit 29 people based on Cobb's Angle range Mild and is included in $\left(1^{\circ} \sim 19^{\circ}\right)$, it can be seen that one person is included in the Moderate $\left(20^{\circ} \sim 39^{\circ}\right)$. Figure 9 is tabulated experimental data.

\section{Conclusion}

In this study, developed scoliosis diagnostic system, is exposed to the radiation is a disadvantage of radiography ah 1 by using an infrared camera and the optical markers can be the diagnosis body safe way there were. By using an infrared camera for diagnosis, and to manufacture a software so that it can be output to the screen, the software, the camera output to the output screen, the angle measuring unit, in consideration of Cobb's Angle measuring unit, I studied the diagnostic algorithm. Experimental Results Male 17 people of this study, the target subject 13 females, a result of measuring the Cobb's Angle value that is diagnosis of scoliosis, 30 hit 29 people based on Cobb's Angle range Mild and is included in $\left(1^{\circ} \sim 19^{\circ}\right)$, it can be seen that one person is included in the Moderate $\left(20^{\circ}\right.$ $\sim 39^{\circ}$ ). Future scoliosis as well as can be expected to accurately measure the spine disease symptoms such as spinal kyphosis.

\section{Acknowledgment}

This study was funded by the Catholic Kwandong University.

\section{References}

[1] M. Agrawal and L.S. Davis, Camera calibration using spheres: A semi-definite programming approach, Proceedings of Ninth IEEE International Conference on System and Computer Vision 2 (2003), 782-789.

[2] R.A. Dickson, Conservative treatment for idiopathic scoliosis, The Journal of Bone and Joint Surgery 67B (1985), 176181 .

[3] P. Beardsley, D. Murray and A. Zisserman, Camera calibration using multiple images, Lecture Notes in Computer Science $\mathbf{5 8 8}$ (1992), 312-320.

[4] L. Berthouze, F. Chavand and C. Barret, A camera neural model, IEEE International Conference on System, Man and Cybemetics, Beijing, 1996, 2124-2127.

[5] J. Black and T. Ellis, Multi camera image tracking, Image and Vision Computing 24 (2006), 1256-1267.

[6] G. Torell, A. Nordwall and A. Nachmson, The changing pattern of scoliosis treatment due to effective screening, Journal of Bone \& Joint Surgery 63 (1981), 337-341.

[7] Stig Willner, Moiré topography for the diagnosis and documentation of scoliosis, The Journal of Acta Orthopaedica. Scand. 50 (1979), 295-302.

[8] S. Brandt and J. Heikkonen, Multi-resolution matching of uncalibrated images utilizing epipolar geometry and its uncertainty, International Conference on Image Processing 2 (2001), 213-216.

[9] Crawford H. Alvin, Strategic directions: The scoliosis research society initiatives for change, Spine 27 (2002), 19601964.

[10] O.D. Faugeras and G. Toscani, Camera calibration for 3D computer vision, Proceedings of International Workship on Industrial Applications of Machine Vision and Machine Intelligence, Silken, 1987, pp. 240-247.

[11] O.D. Faugueras and G. Toscani, The calibration problem for stereoscopic vision, Sensor Devices and Systems for Robotics F52 (1989), 195-213.

[12] K. Allen Greiner, Adolescent idiopathic scoliosis: Radiologic decision-making, American Family Physician 65 (2002), 1817-1823. 